\title{
Effect of the use of ambulance-based thrombolysis on time to thrombolysis in acute ischemic stroke: a randomized clinical trial
}

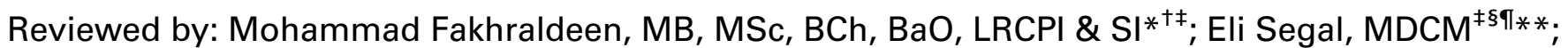 \\ François de Champlain, $\mathrm{MD}^{\ddagger \S}$
}

\begin{abstract}
Article chosen
Ebinger M, Winter B, Wendt M, et al. Effect of the use of ambulance-based thrombolysis on time to thrombolysis in acute ischemic stroke: a randomized clinical trial. JAMA 2014;311(16):1622-31.

Clinical question

Does prehospital thrombolysis in specialized ambulances reduce delay to thrombolysis in acute ischemic stroke?

Objective

To determine the effect of prehospital thrombolysis for acute ischemic stroke administered in specialized ambulances on delay in thrombolytic administration, thrombolysis rate, post-thrombolysis intracerebral hemorrhage, and 7-day mortality.
\end{abstract}

Keywords: Prehospital, stroke, thrombolysis, reperfusion delays, mobile unit

\section{BACKGROUND}

Thrombolysis is one of few treatments available for acute ischemic stroke; however, its use remains controversial. ${ }^{1}$ The American Heart Association guidelines $^{2,3}$ and a recently released draft position statement from the Canadian Association of Emergency Physicians (CAEP) ${ }^{4}$ both recommend recombinant tissue-type plasminogen activator (rTPA) for acute ischemic stroke. However, many emergency physicians remain unconvinced of the benefit of thrombolysis for stroke in light of its potential for serious adverse events (e.g., increased risk of intracranial hemorrhage and early mortality). Emergency physicians who have questioned the recommendations made in CAEP's draft position statement have suggested that a reconsideration of the quality of the evidence is necessary before CAEP embraces thrombolysis in this scenario. Controversy aside, even if its use were universally advocated, achieving timely treatment has been challenging.

A previous randomized controlled trial ${ }^{5}$ comparing prehospital stroke treatment using mobile stroke units to standard hospital stroke care found substantial time reductions from activation to therapeutic decision.

This study, the prehospital acute neurological treatment and optimization of medical care in stroke study (PHANTOM-S), ${ }^{6,7}$ was preceded by a 3 -month pilot study to assess the stroke emergency mobile unit (STEMO) deployment feasibility and safety. This special ambulance carried special equipment (a computed tomography $[\mathrm{CT}]$ scanner, point-of-care laboratory, and teleradiology system) and three staff (a paramedic, a neurologist, and a radiology technician).

\section{Study design}

This was an open-label, randomized-week clinical trial conducted in Berlin, Germany between May 1, 2011 and January 31, 2013. Weeks were randomized to STEMO availability or routine care to assess the effectiveness of prehospital tissue plasminogen activator $(\mathrm{tPA})$ in improving call-to-needle time.

From the *Royal College of Surgeons in Ireland, National University of Ireland, Dublin; †Trinity College, Dublin; $\neq$ Department of Emergency Medicine, McGill University Health Centre, Montreal, OC; §Department of Family Medicine, McGill University, Montreal, QC; IDepartment of Prehospital Medicine, Sacre-Coeur Hospital, Montreal, QC; and **Urgences-santé, Montreal, OC.

Correspondence to: Mohammad Fakhraldeen, Emergency Medicine Residency Training Program, Room A4.62, Royal Victoria Hospital, 687 Pine Avenue West, Montreal, Quebec H3A 1A1; Email: mohammad.fakhraldeen@mail.mcgill.ca 


\section{Population}

The study included nonpregnant patients at least 18 years old with a symptom onset that was within 4 hours of the activation. Independent of STEMO availability, stroke dispatch activation occurred between 0700 hours and 2300 hours only.

\section{Outcomes measured}

The primary outcome was duration from emergency dispatcher activation to tPA administration. Secondary outcomes were thrombolysis rate, post-tPA intracerebral hemorrhage, and mortality.

\section{RESULTS}

During the 21-month study period, there were 7098 stroke activations: 3668 during STEMO weeks and 3430 during control weeks. During STEMO weeks, 1641 (45\%) activations did not lead to STEMO deployment because the STEMO was already in operation or undergoing maintenance. Of patients with in-hospital documented ischemic strokes, investigators excluded those who did not receive intravenous $\mathrm{PPA}$, received MRIbased tPA, were enrolled in parallel thrombolysis studies, or had no neurological deficit. In total, 518 patients received tPA and were included in the primary analysis, exceeding the predetermined sample size requirement (456) to detect a 20-minute intergroup difference.

Patients were analysed in three groups (Tables 1 and 2): STEMO deployment, STEMO weeks (including patients with and without STEMO deployment), and control weeks.

Alarm-to-treatment time was 15 minutes (95\% confidence interval [CI] 11-19 minutes) shorter during STEMO versus control weeks. Other notable findings in the STEMO deployment group included a larger proportion of patients treated within 90 minutes of symptom onset $(57.5 \%$ [95\% CI $50.6-64.1]$ v. $37.4 \%$ [95\% CI 31.3-44.0], respectively) and a 16-minute shorter onset-to-treatment time (102.7 minutes [95\% CI $93.9-111.5$ ] v. 118.5 minutes [95\% CI $111.8-125.2$ ], respectively).

\section{COMMENTARY}

This study addressed one of the main factors in the management of hyperacute stroke with thrombolysis: prehospital delay. Baseline patient characteristics were similar in both groups, except for slightly higher rates of atrial fibrillation (AF) and diabetes mellitus (DM) in the STEMO deployment group. Although AF and DM are associated with higher post-stroke mortality and more severe functional deficits, ${ }^{8-10}$ there was no significant difference in secondary outcomes between the two groups. There was also a notably larger proportion of patients with higher stroke severity scores (National Institutes of Health Stroke Scale [NIHSS] scores $\geq 20$ ) in the STEMO deployed (12\%) versus the control group (6\%), albeit without an impact on outcome. NIHSS describes stroke-related neurological deficits, and strokes with scores above 20 are considered very severe.

One important finding of this study is the apparent safety of the intervention: STEMO care was found to be non-inferior to conventional stroke care in terms of secondary intracerebral hemorrhage and 7-day mortality. Conceivable harms of this novel treatment approach do not appear to be different from those of current practice.

Patients were analysed using an intention-to-treat approach (comparing all patients in intervention and control weeks in their respective randomized groups) and a per-protocol analysis (comparing patients who were actually treated in STEMO to those receiving regular prehospital care). In keeping with their intention-to-treat approach, the authors analysed patients enrolled during

Table 1. Alarm-to-treatment times and thrombolysis rates

\begin{tabular}{|c|c|c|c|}
\hline \multirow[b]{2}{*}{ Outcome measure } & \multicolumn{2}{|c|}{ STEMO* weeks } & \multirow[b]{2}{*}{ Control weeks } \\
\hline & All patients & Patients with STEMO deployment & \\
\hline Mean alarm to treatment time, ${ }^{a}$ minutes $(95 \% \mathrm{Cl})$ & $61.4(58.7-64.0)$ & $51.8(49.0-54.6)$ & $76.3(73.2-79.3)$ \\
\hline Thrombolysis rates ${ }^{\mathrm{b}}$ & $29 \%(310 / 1070)$ & $33 \%(200 / 614)$ & $21 \%(220 / 1041)$ \\
\hline
\end{tabular}


Effect of the use of ambulance-based thrombolysis on time to thrombolysis in acute ischemic stroke

Table 2. Secondary intracerebral hemorrhage and seven-day mortality

\begin{tabular}{lcc}
\hline Outcome measure & Patients with STEMO deployment & Patients without STEMO deployment \\
\hline Post-tPA intracerebral hemorrhage $^{\mathrm{a}}$ & $3.5 \%(7 / 200)$ & $6.8 \%(22 / 323)$ \\
Seven-day mortality $^{\mathrm{b}}$ & $4.5 \%(9 / 199)$ & $4.6 \%(15 / 323)$ \\
\hline
\end{tabular}

aThere was no significantly increased risk of secondary intracerebral hemorrhage in the STEMO deployment group compared to patients who did not experience STEMO deployment (this latter group comprises patients in the STEMO group who did not experience STEMO deployment in addition to patients in the control group); adjusted odds ratio (OR) 0.42 (95\% CI 0.18-1.03, $\mathrm{p}=0.06)$.

${ }^{\mathrm{b}} \mathrm{STEMO}$ deployment was not associated with a significantly increased risk of seven-day mortality; adjusted OR $0.76(95 \% \mathrm{Cl} 0.31-1.82, \mathrm{p}=0.53)$.

STEMO weeks who did not receive STEMO treatment and patients who received STEMO treatment but received hospital-based tPA in the STEMO deployment group. Applying this effectiveness approach reflects the unpredictable reality of emergency medicine, making the results more generalizable than those of an efficacy trial. A closer look at the results of the intention-to-treat analysis reveals that only $29 \%$ of the STEMO group patients received $\mathrm{tPA}$, with an average time saved in delay to treatment of approximately 15 minutes.

The potential time-sensitive benefit of $\mathrm{tPA}$ in ischemic stroke formed the basis for the primary outcome: alarmto-treatment time, which was substantially shorter in the STEMO deployment group. However, this measure does not necessarily translate into a clinically relevant patient benefit. An accompanying editorial suggested that reducing time to treatment may not yield clinical improvement. ${ }^{1}$ The ongoing controversy about tPA for stroke may also impact readers' interpretations of the study results. Firm believers in tPA would likely argue for bringing this intervention faster and to a greater number of patients. However, tPA critics would likely emphasize the study's lack of significant improvements in patient-oriented outcomes.

\section{STEMO in Canada?}

Despite continued controversies regarding the role of tPA in acute stroke, earlier treatment is preferred once the decision to thrombolyse is made, and thus the observed reduction in onset-to-needle time may be argued to support the introduction of such services in Canada.

Despite the validity of the results, the generalizability of the findings of this study to the current situation in Canada is questionable. The cost-effectiveness of this intervention would need to be rigorously evaluated, and the authors indicate that such analyses are currently underway. The cost of this study's intervention lies both in the STEMO unit and in the resource-intensive personnel training and ongoing staffing of a mobile unit that includes advanced, specialized human resources. Such a model has poor external validity in a North American context where prehospital care is provided almost exclusively by paramedics. In addition, whether such a model would be cost-effective in Canadian urban and rural settings would also need to be evaluated.

While earlier thrombolysis may be appealing in the context of "time is brain," this study's intervention is definitely not something we would expect to see in Canada in the near future. Early prehospital thrombolysis for stroke has yet to be definitively proven to improve patient-oriented functional outcomes in prospectivecontrolled trials. ${ }^{1}$ In addition, before investing precious resources in such a program, the results of ongoing cost-effectiveness analyses must be reviewed. This costly resource should also be contrasted with the opportunity cost of not pursuing other strategies that could diminish treatment delays, such as public stroke campaigns for early symptom recognition, telemedicine, and improvements in the rapidity of emergency department assessment. A good example is the successful application of the Helsinki model to minimize in-hospital delays to tPA in Melbourne. ${ }^{11}$

\section{CONCLUSION}

Ambulance-based thrombolysis decreased the time to treatment compared with conventional stroke care without increasing the 7-day mortality or the incidence of intracerebral hemorrhage. The cost-effectiveness and wider applicability of this intervention needs further assessment, including its impact on patient-oriented outcomes.

Competing interests: None declared.

\section{REFERENCES}

1. Grotta JC. tPA for stroke: important progress in achieving faster treatment. FAMA 2014;311(16):1615-7. 
2. Adams HP Jr, del Zoppo G, Alberts MJ, et al. Guidelines for the early management of adults with ischemic stroke: a guideline from the American Heart Association/American Stroke Association Stroke Council, Clinical Cardiology Council, Cardiovascular Radiology and Intervention Council, and the Atherosclerotic Peripheral Vascular Disease and Quality of Care Outcomes in Research Interdisciplinary Working Groups. Circulation 2007;115:e478-534.

3. Lees KR, Bluhmki E, von Kummer R, et al. Time to treatment with intravenous alteplase and outcome in stroke: an updated pooled analysis of ECASS, ATLANTIS, NINDS, and EPITHET trials. Lancet 2010;375(9727):1695-703.

4. Rutledge $T$, Bergmann $T$, Cass $D$, et al. The CAEP Committee on Thrombolytic Therapy for Acute Ischemic Stroke). Thrombolytic therapy for acute ischemic stroke. Available at: http://caep.ca/resources/position-statements-and-guidelines/thrombolytic-therapy-acute-ischemic-stroke (accessed October 16, 2014).

5. Walter S, Kostopoulos P, Haass A, et al. Diagnosis and treatment of patients with stroke in a mobile stroke unit versus in hospital: a randomized controlled trial. Lancet Neurol 2012;11(5):397-404.
6. Ebinger M, Rozanski M, Waldschmidt C, et al. STEMOConsortium. PHANTOM-S: the prehospital acute neurological therapy and optimization of medical care in stroke patients study. Int 7 Stroke 2012;7(4):348-53.

7. Weber JE, Ebinger M, Rozanski $M$, et al. STEMOConsortium. Prehospital thrombolysis in acute stroke: results of the PHANTOM-S pilot study. Neurology 2013;80 (2):163-8.

8. Gustasson C, Britton M. Pathogenetic mechanism of stroke in non-valvular atrial fibrillation: follow-up of stroke patients with and without atrial fibrillation. $\mathcal{F}$ Intern Med 1991;230(1):11-6.

9. Lin HJ, Wolf PA, Kelly-Hayes M, et al. Stroke severity in atrial fibrillation. The Framingham Study. Stroke 1996;27: 1760-4.

10. Tuomilehto J, Rastenyte D, Jousilahti P, et al. Diabetes mellitus as a risk factor for death from stroke. Prospective study of the middle-aged Finnish population. Stroke 1996;27:210-5.

11. Meretoja A, Weir L, Ugalde M, et al. Helsinki model cut stroke thrombolysis delays to 25 minutes in Melbourne in only 4 months. Neurology 2013;81(12):1-6. 